\title{
Itinéraires Itinéraires
}

Littérature, textes, cultures

2019-1 | 2019

La « renaissance littéraire » africaine en débat

\section{À quel prix vêt-on la terre ou le vent ? Regards mulongo sur le vêtement dans La Saison de l'ombre de Léonora Miano}

At What Price Can Earth or Wind Be Clothed? Looking at Clothing through

Mulongo Eyes in La Saison de l'ombre, by Léonora Miano

Pierre-Yves Dufeu

\section{OpenEdition}

Journals

Édition électronique

URL : http://journals.openedition.org/itineraires/5805

DOI : 10.4000/itineraires.5805

ISSN : 2427-920X

Éditeur

Pléiade

Référence électronique

Pierre-Yves Dufeu, « À quel prix vêt-on la terre ou le vent ? Regards mulongo sur le vêtement dans $L a$ Saison de l'ombre de Léonora Miano », Itinéraires [En ligne], 2019-1 | 2019, mis en ligne le 23 juillet 2019 consulté le 02 août 2019. URL : http://journals.openedition.org/itineraires/5805 ; DOI : 10.4000/ itineraires.5805

Ce document a été généré automatiquement le 2 août 2019

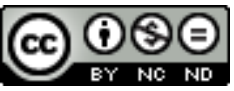

Itinéraires est mis à disposition selon les termes de la licence Creative Commons Attribution - Pas d'Utilisation Commerciale - Pas de Modification 4.0 International. 


\title{
À quel prix vêt-on la terre ou le vent ? Regards mulongo sur le vêtement dans La Saison de l'ombre de Léonora Miano
}

\author{
At What Price Can Earth or Wind Be Clothed? Looking at Clothing through \\ Mulongo Eyes in La Saison de l'ombre, by Léonora Miano
}

Pierre-Yves Dufeu

1 Récit après récit, Léonora Miano sait émouvoir, toucher avec talent un public universel, tout en donnant vie, de façon très documentée, à des groupes humains particuliers, notamment ceux issus de ce pays qu'on appelle aujourd'hui Cameroun ${ }^{1}$. Dans La Saison de l'ombre, récit paru en 2013, elle choisit de poser la question de la traite négrière du point de vue d'une communauté particulière, les Mulongo, qui, vivant dans la forêt éloignée des côtes, en subit les effets sans les comprendre. Elle déploie à partir de là un genre littéraire original, qui a pu être défini comme roman policier historique (Mokam 2015). Elle place ainsi un personnage de femme mulongo en situation de sortir du village, de suivre les traces des disparus, d'atteindre cette fin du monde qu'est pour elle la côte et d'y faire bien des découvertes inattendues. L'une des forces de ce récit, c'est que le lecteur du xxI siècle est à la fois parfaitement conscient de ce qui se passe, la capture de jeunes mulongo qui ont probablement été vendus comme esclaves et emportés par bateau vers les Amériques, et à la fois, par le jeu de l'empathie narrative, émotionnellement solidaire de ce personnage principal qui cherche, ne comprend pas, et découvre peu à peu la vérité sous l'aspect de ses propres références culturelles. On peut y voir une mise en écriture mais surtout en lecture, de la «double conscience » que les théoriciens du $\mathrm{xx}^{\mathrm{e}}$ siècle $^{2}$ ont pu mettre au jour, mais ici pour rendre compte du destin de communautés africaines anciennes et sur un plan fictionnel.

2 Nous voudrions montrer comment, dans ce récit à forte résonance historique, symbolique et interculturelle, la perception du vêtement joue un rôle subtil dans la révélation de 
cette vérité au cœur du drame des Mulongo. Les anthropologues ${ }^{3}$ ont pu établir que le vêtement remplit des fonctions de protection, de signification et d'esthétique ${ }^{4}$. Pour Leroi-Gourhan, « le vêtement est l'instrument de la dignité de l'homme », formule qui fait très heureusement la synthèse entre le rôle pratique et le rôle sémantique, voire spirituel d'un objet qu'on aurait donc tort de réduire à sa seule épaisseur matérielle. L'usage fictionnel de ce référent est en outre révélateur d'une ontologie (Descola 2005), celle des communautés fictives mais vraisemblables mises en scène. En le mentionnant, en le décrivant dans son récit, Léonora Miano ne sacrifie pas seulement aux exigences du réalisme documentaire: elle signifie toujours quelque chose, dans l'économie du récit même, et dans cette avancée de l'ombre qu'évoque le titre du roman. Le vêtement n'est pas seulement dans ce récit un élément statique de description : lié à d'autres référents, il intervient toujours comme le signe d'une dynamique tragique que porte le récit, figure de l'histoire universelle. Il est toujours l'objet d'un regard subjectif, culturellement déterminé, dans l'économie complexe de la coexistence de ces communautés anciennes contemporaines des siècles classiques de la traite négrière. C'est là que la puissance thématique du récit lui donne aussi une dimension politique et, en congruence avec les réflexions théoriques exactement contemporaines d'un Achille Mbembe ${ }^{5}$ par exemple, invite à réfléchir, à travers l'invention du « nègre ${ }^{6}$ » à l'abolition, par un pouvoir invisible, de la frontière entre chose et personne. Dans ce contexte, le vêtement qu'engage la fiction romanesque y apparaît autant comme signe ou indicateur de la «fongibilité » (Mbembe 2013 : 16) de l'humain lui-même que comme instrument de sa dignité. La mention toute littéraire de cet objet si humain, le vêtement, invite ainsi à considérer aussi l'esclavage du point de vue de ces objets inanimés auxquels l'esclave, ce nègre pressenti et reconnu tel par le lecteur au terme du récit, est paradoxalement assigné.

3 Le récit de La Saison de l'ombre est conduit en focalisation zéro à forte tendance interne : la narration rend compte, à la troisième personne, des faits, gestes, paroles extérieures, mais ne le fait jamais sans s'approcher tour à tour du personnage qu'elle suit, au point de laisser percevoir son regard, sa pensée, sa conscience. Or, tous les personnages ainsi approchés dans le récit par cette singulière narration empathique sont mulongo, ils appartiennent tous à ce peuple des forêts. Ce choix narratif facilite l'empathie du lecteur lui-même à l'égard de cette petite société africaine, qu'il découvre pourtant au fur et à mesure. Le récit rend compte en particulier de l'initiation d'Eyabe, personnage féminin principal, et, à travers elle, laisse percevoir au lecteur la profondeur historique et anthropologique de ces territoires africains antérieurs à la colonisation européenne. Superficiel par nature, le vêtement et ses discrètes variations livrent ainsi subtilement une profondeur inattendue, ils nous révèlent cette superposition de communautés qui seule explique in fine ces disparitions, objet de la quête d'Eyabe. Les mentions du vêtement, ou de l'étoffe - car, pour un Mulongo, celle-ci correspond nécessairement à celui-là - constituent ainsi un discret fil d'Ariane cousu dans le tissu symbolique du récit, fil qui s'enrichit ou s'épaissit au fur et à mesure de son avancée. L'exploitation littéraire des fonctions anthropologiques du vêtement évolue au fil du récit. On peut ainsi distinguer, dès les premières pages, une conception surtout pratique du vêtement, celle de la communauté mulongo, associée notamment à la force et à la résilience des femmes et reliée à la fonction de protection qui est aussi celle de l'écorce végétale dont il est tiré. Dans son deuxième chapitre, le récit évoque et souligne l'étonnement d'un personnage mulongo face aux tissus de la communauté bwele voisine et à l'art de leur confection, les fonctions sémiotique et symbolique (Delaporte 1990: 970-997) du vêtement sont alors questionnées et par là mises en exergue. Enfin, sont très tôt mentionnées d'étranges 
étoffes imprimées venues d'au-delà des eaux et associées à une communauté d'étrangers : leur fonction esthétique saillante leur conférera, au-delà même du récit mais visé par lui, un pouvoir inouï.

\section{Vêtements des femmes et de la forêt : force de l'écorce et de l'étoffe}

Dans le récit, les femmes apparaissent d'emblée au centre de la communauté mulongo. Elles sont évoquées les premières, et ce sont, autant que leur peine oubliée ou refoulée ${ }^{7}$, leurs grandes qualités de force morale, de résilience ${ }^{8}$, que l'auteur entend d'abord mettre en exergue. Les vêtements décrits sont donc d'abord des vêtements de femmes, et ils vont signifier à leur façon toutes ces qualités, car ils sont aussi le produit de leur travail :

Elles n'échangeront que des paroles banales, celles qu'on dit en exécutant les tâches domestiques. Les mots que l'on prononce lorsqu'on pile des tubercules à deux. Quand on rassemble des fibres végétales pour confectionner un dibato ou une manjua. (Miano 2013 : 17)

Le récit ne fait pas ici que rapporter le vêtement mulongo au travail des femmes, il le relie, lui et ses noms, à la parole des femmes, comme si ces « paroles banales » féminines étaient en fait le tissu du quotidien de la communauté, celui qui lui permet de vivre, de se nourrir, de se vêtir. Le vêtement apparaît ainsi, dans la communauté mulongo, comme le produit de la parole créatrice et collective de ces femmes. En ce sens, l'énallage, le passage de «elles» à "on» est significatif, il renforce le caractère empathique de la narration et fait également de ces femmes le centre effectif de leur communauté9.

Chez les Mulongo, le vêtement appartient donc toujours d'abord aux femmes au sens où ce sont les femmes qui l'auront confectionné. Le texte mentionne deux noms traditionnels, "dibato » et "manjua », dont le glossaire douala annexé au récit fournit des définitions :

Dibato (pluriel : mabato) : étoffe

Manjua : vêtement à franges, en fibres végétales, qui ressemble à une jupe ; se porte

en signe de lamentation. (Miano 2013 : 243)

Cette distinction entre les deux habits mulongo est exploitée assez tôt dans le récit, dont le propos est volontiers didactique :

Eyabe pénètre maintenant dans la case. Elle fredonne une complainte, tape doucement des mains.

Bientôt, elle ressort, vêtue d'un dibato en écorce battue. C'est un costume

d'apparat, pas comme la manjua. Eyabe se dirige vers le centre du village, avance lentement. (Miano $2013: 26$ )

Le lecteur, s'il est peu familier de ces cultures, comprend ainsi que les deux vêtements sont confectionnés à partir de fibres ou d'écorces végétales. Les Mulongo tirent naturellement leurs vêtements de la forêt dans laquelle ils vivent. Le passage ci-dessus nous rappelle en outre que l'écorce, devenue étoffe, donne force. La fonction de protection du vêtement s'inscrit ainsi parfaitement dans une anthropologie analogique, et non naturaliste ${ }^{10}$, en laquelle l'affinité extérieure arbre / homme est construite par la transformation de l'écorce en seconde peau de protection au sein de l'univers mulongo. Les femmes mulongo, leur travail et leur parole confectionnent donc, autant que ces vêtements, l'analogie qui les rend possibles dans leur fonction de protection. Cette fonction du vêtement apparaît ainsi saillante dans cet univers fictif mulongo, mais jamais unique, comme nous l'apprennent les anthropologues et comme l'illustre ici Léonora 
Miano à travers l'évocation différentielle du dibato, « costume d'apparat » : la fonction sociale et symbolique est donc bien présente elle aussi, sur un mode mineur.

Quelques pages auparavant, Eyabe s'est détachée du groupe indistinct des mères de disparus, condamnées à l'isolement par le village. Le récit lui a accordé un nom individuel. Voici qu'à travers le dibato, l'écorce battue, elle se revêt elle-même de force et de solennité pour briser cet isolement qui pèse à toutes, en demander raison à la communauté.

Les vêtements mulongo, tirés des ressources de la forêt, portent en eux la parole, la force créatrice des femmes. Des femmes capables de se suffire à elles-mêmes :

Pendant votre séjour ici, vous ne recevrez pas de viande. Tout ce dont vous aurez besoin est là... Une de mes coépouses a rassemblé des étoffes et des fibres dont vous ferez vos vêtements, vos nattes. (Miano 2013 : 38)

C'est l'ancienne du village qui parle ici aux mères des douze disparus. Tout le premier chapitre du récit met constamment en valeur la force, la résilience de ces femmes, à travers cette épreuve sociale et d'abord émotionnelle de la perte du fils, puis de la mise à l'écart de la communauté. Dans cette épreuve, le vêtement apparaît toujours à la fois comme le signe et le produit de leur constance, de leur patience qui est la vie même, en lien avec cette nature végétale toute proche qui leur procure les matières dont elles se vêtent ou vêtent la communauté tout entière.

Dans la suite du récit, le vêtement féminin mulongo ne sera plus associé qu'à Eyabe. Tiré de la forêt, fait de la main des femmes et lié à leur parole, il soulignera dans le récit à la fois le destin et la force de ce personnage féminin, qu'il alourdisse ses épreuves ou au contraire l'en protège, conformément à la première de ses fonctions anthropologiques :

Eyabe tente de ne pas s'affoler. Il faut garder confiance. La fatigue la gagne. Elle avance. La boue lui couvre les mollets, alourdit les franges de sa manjua, mais elle avance. (Miano 2013 : 119)

Une vieille impotente est assise devant l'une des demeures. Les voyant passer, elle marmonne quelque chose d'inaudible, envoie un long crachat dans leur direction. Le jet glaireux s'écrase au sol, manque de peu les passants. Eyabe détourne les yeux, resserre les pans de l'étoffe qui lui couvre les épaules. (Miano 2013 : 175)

Cette ambivalence de l'habit mulongo figure celle du parcours d'Eyabe, dont l'âme devient à la fois plus lourde et plus solide au fur et à mesure de ses découvertes hors du territoire familier. L'occultation progressive, dans la narration, de la fonction de protection du vêtement figure la prise de conscience par cette communauté mulongo du passage d'une ère de protection à la saison de l'ombre, lourde d'inconnu et de menaces.

\section{Mutango face aux tissus bwele}

Dans le deuxième chapitre du roman, «Dires de l'ombre », la narration empathique du récit se rapproche d'un personnage mulongo masculin. Mutango, frère jaloux et adipeux du chef du village, s'éloigne du territoire mulongo jusqu'à parvenir chez les Bwele, le peuple voisin. C'est donc à travers ses yeux que nous découvrons la société bwele, voisine mais pour lui étrange, étrangère. Se déploient alors les développements les plus longs que consacre le récit à la thématique du vêtement. Cette longueur est en soi un indice de la dimension symbolique et nécessaire de ce référent dans le récit, d'autant plus qu'elle nourrit précisément une réflexion sur ses fonctions esthétique puis socio-sémantique :

Le gros regarde aussi les vêtements des rares personnes attardées là, la finesse $d u$ travail des artisans bwele qui font des merveilles avec leurs métiers à tisser l'esoko. 
Chez lui, les étoffes les plus délicates ne sont que battues, ce qui ne fait pas appel à la même ingéniosité. Mutango se sent tout à coup un peu arriéré, se fait l'effet d'un loqueteux. [...] Il se rassure en se disant que, malgré tout, les Mulongo sont plus habiles à travailler les peaux. Les chefs de sa communauté possèdent, pour en attester, un habit de cérémonie en léopard, appelé mpondo. Ce costume est si beau qu'il confère prestance, autorité, à celui qui le revêt. Même lorsqu'il s'agit d'un individu aussi insignifiant que son frère Mukano. Un jour prochain, l'homme en est certain, il revêtira le mpondo, tiendra d'une main ferme le bâton de commandement. (Miano 2013 : 93)

La finesse du tissage bwele laisse apparaître à Mutango comme au lecteur une subtile frontière culturelle. Les Bwele savent tisser, quand les Mulongo ne savent qu'assouplir, en les battant, les ressources de la forêt, fibres, écorces. Mais l'admiration spontanée ne dure pas, elle laisse vite la place chez Mutango à deux passions basses, tout d'abord une certaine honte, puis une survalorisation, par l'imagination, du vêtement le plus prestigieux à ses yeux, fait de la peau du léopard, ce souverain de la forêt. La vanité et cette jalousie qui s'exerce autant à l'encontre de son frère que des Bwele, suspendent en Mutango ses capacités d'attention, au bénéfice d'une rêverie mesquine et masculine ${ }^{11}$ en laquelle pouvoir et vêtement en viennent à se confondre. Le lecteur parvient ainsi moralement aux antipodes du regard naturel, explicite et collectif que portent les femmes mulongo sur les vêtements d'origine végétale qu'elles confectionnent et qui mettait en avant leur fonction de protection. À partir de l'étoffe mulongo familière, faite de fibre ou d'écorce, et première dans le récit, se tisse ainsi, dans ce passage, un réseau d'oppositions, bwele / mulongo, battre / tisser, homme / femme, animal / végétal qui démultiplie les formes et les rapports au vêtement. Or, ces oppositions se conjuguent d'une façon inattendue :

En passant devant un tisserand occupé à ranger son matériel, le dignitaire s'arrête, observe l'outil qui permet la fabrication du tissu en esoko. Puis, quelque chose le frappe, l'indigne. Il s'adresse à Bwemba: Ce sont donc les hommes qui effectuent ce travail? Son compagnon acquiesce, précise: Autrefois, la fonction se transmettait uniquement entre mâles d'une même famille. De nos jours, c'est un simple métier, qu'on apprend auprès d'un maître. Celui-ci met ses choses en ordre parce que la nuit arrive. Il est interdit de tisser après le départ du soleil. Mutango distingue mal les parties du métier à tisser, tente d'évaluer la pénibilité d'une activité qui, pour lui, devrait échoir aux femmes. Un mâle a mieux à faire. (Miano 2013 : 94)

Étrangeté fondamentale pour Mutango, le tissu bwele ne vient ni de la forêt, ni du travail des femmes. Comme Ahmadou Kourouma avant elle ${ }^{12}$, Léonora Miano, dont le territoire national s'enorgueillit encore aujourd'hui du nombre de ses ethnies ${ }^{13}$, interroge ainsi la question des frontières culturelles internes à l'Afrique. Et, contrairement à Kourouma, plus léger, fantaisiste, volontiers humoristique dans son approche de cette question, elle le fait sur le mode restreint et mesquin qu'implique son choix d'une narration empathique ici liée à un personnage antipathique. Elle engage ainsi une critique implicite des schémas de domination genrée qui déterminent la perception de Mutango. Mais elle démonte aussi à travers lui, plus généralement, les mécanismes du préjugé culturel, de l'ostracisme, qui est aveuglement, fuite du réel au profit du fantasme, décision implicite de mépriser plutôt que d'apprécier : Mutango « distingue mal » ce qui sert à tisser parce que déjà dans son esprit cette activité tout entière, quelque fin qu'en soit le produit, est dévaluée par l'indignation qu'il éprouve, dont il n'entend pas sortir et qui lui tient lieu de regard. Ainsi toutefois pensé comme un objet social plutôt que d'abord pratique, le vêtement, loin de protéger, inquiète en ce qu'il révèle un nouvel ordre symbolique. 
Cette frontière entre les deux cultures apparaît plus profondément encore lorsque Mutango observe de l'intérieur la demeure de la reine bwele :

Les serviteurs alignent les tabourets à caryatide sur lesquels d'éminentes personnalités prendront place, déroulent, entre les deux rangées de sièges, une toile en esoko qui a la particularité d'être brodée par les femmes bwele. Le notable mulongo s'étonne de les voir déplier un tissu à même le sol. Le chasseur lui dit que ce textile n'est pas conçu pour l'habillement. Cette étoffe, tissée comme toutes les autres par les hommes, est considérée à la fois comme un élément du mobilier et comme un objet décoratif. Une fois façonnée par les tisserands, elle est confiée à une catégorie précise de femmes - il ne dit pas laquelle - chargées d'y broder des motifs selon leur fantaisie.

L'homme n'est pas certain de tout saisir : d'où il vient, les artisans sont, bien sûr, très attachés à la beauté de leurs œuvres, mais elle est conditionnée par l'adéquation entre l'objet et sa signification profonde. C'est précisément ce qui lui échappe ici : le sens. Sans le questionner davantage, il songe que son interlocuteur ne lui dit pas tout, continue d'observer ce tissu créé pour que l'on marche dessus, comme si la terre n'était pas assez bonne, comme s'il n'était plus absolument primordial d'entretenir avec elle des liens puissants. Lorsque le chasseur ajoute que l'étoffe en question est aussi utilisée pour envelopper les corps des nobles avant leur mise en terre, le gros homme ne cherche plus à comprendre. On ne peut consacrer le même matériau à des usages si différents, c'est absurde. (Miano 2013 : 95-96)

$\mathrm{Nul}$ besoin de souligner combien les fonctions anthropologiques sémiotique et symbolique (Delaporte 1990 : 970-997) de l'étoffe sont ici explicitement mises en valeur. Ce passage illustre parfaitement l'art qu'a Léonora Miano de creuser la distance entre la connaissance extérieure de son lecteur et celle, plus restreinte ici, de son personnage, de mettre en lecture la double conscience ${ }^{14}$. La focalisation sur Mutango ici adoptée permet de percevoir à travers lui, de s'étonner avec lui, et, au point de rupture interculturel, sinon de ne pas comprendre, puisque le lecteur reconnaît un tapis dans cet objet bwele ici non désigné, de faire du moins l'effort de comprendre l'incompréhension de Mutango.

Or cette incompréhension même est signifiante, et l'auteur pousse assez loin cette réflexion : que signifie un tel objet, que peut signifier, pour un Mulongo, vêtir la terre? L'étoffe mulongo protège le corps comme l'écorce l'arbre dont elle est tirée. Mais que protège une étoffe déployée à terre ? Mutango d'ailleurs, sans s'y attarder, entrevoit le prix de cette subversion de l'usage : bien loin de protéger, une telle pratique menace de rompre la communication sensuelle, organique, de l'homme avec la terre, puisque, ainsi séparé par l'étoffe, son pied n'est plus cette racine mobile qui, la foulant, la ressent aussi. Pour des raisons obscures, les Bwele isolent donc l'homme de la terre et de la forêt nourricières : ils vêtent son corps, non de fibres ou d'écorces, mais d'esoko tissé, ils éloignent son pied du contact avec la terre. Une fonction symbolique inconnue fait donc disparaitre la fonction de protection.

La peur de Mutango n'est pas dite. Elle est toutefois symbolisée, et, comme toujours chez ce personnage peu curieux et présomptueux, occultée par un mépris qui exprime surtout sa profonde bêtise. Toutefois, la mention par Bwemba de ce que les Bwele utilisent encore cette étoffe pour vêtir et ensevelir les morts de haut rang est assez éloquente. Si déjà l'étoffe ne protège plus, si elle menace et déséquilibre l'ordre du monde, voilà que les Bwele lui accordent le pouvoir de retenir et d'accompagner ces morts qui, vivants, furent les plus puissants. Dans ces conditions, ainsi détournée de l'usage naturel qu'en indique la forêt, que ne peut-elle pas? Capturer les vivants? Les tuer peut-être? Mutango garde à l'esprit que douze des siens ont disparu, son apparente indifférence ou refus de 
comprendre dissimule une crainte symbolique : si le sens lui échappe, c'est qu'on lui cache un danger.

Or, dans la suite du récit, Mutango, hôte des Bwele, sera précisément invité à quitter son vêtement mulongo pour revêtir un tissu bwele :

Le garçon lui présente ce qui ressemble à une étoffe pliée. C'est lui qui parle le premier, une moue de désapprobation lui abaissant les lèvres: Étranger, je me permettrai de rafraîchir ton... habit demain. Tu le laisseras sur le lit, et revêtiras ceci, fait-il en lui tendant le tissu. (Miano $2013: 101$ )

S'emparant du vêtement qu'on lui a remis, il entreprend de le déplier. L'idée lui vient soudain qu'il ne se ferait pas remarquer, s'il le portait. Quelques-unes des amulettes qui ne le quittent jamais le trahiraient peut-être, mais de loin, on les verrait à peine, dans ce pays où les accoutrements peuvent se révéler d'une rare extravagance.

L'homme se débarrasse de sa manjua, noue l'étoffe autour de son large bassin, réfléchit un instant, décide de ne pas laisser sa gibecière. (Miano $2013: 102$ )

Le garçon bwele cache mal son mépris à l'égard du vêtement mulongo, au point qu'il peine même à le reconnaitre tel. Mépris probable envers la grossièreté de la manjua non tissée mais battue, mais aussi dégoût de l'odeur qui s'en dégage, car Mutango, avant d'arriver chez les Bwele, a dû marcher et dormir longtemps dans la forêt, l'habit frottant constamment contre la gibecière pleine de viande boucanée.

La décision que prend Mutango de revêtir en effet l'étoffe bwele ne répond pas à la suggestion de ses hôtes, dont il se méfie, mais plutôt à une intention de s'échapper pour ne pas rester à leur merci pendant la nuit. Elle prend toutefois une signification symbolique: quittant la manjua pour le tissu bwele, Mutango, dignitaire mulongo, devient de lui-même sujet de la princesse bwele. Hanté par la parole des disparus mulongo, "Ne sais-tu pas que les Bwele ont jeté sur nous leurs filets? ", Mutango croyant fuir enfile une étoffe qui le retiendra mieux qu'un filet. Ainsi revêtu, il ne quittera plus ce monde ; tenu plus tard pour un espion, il y deviendra esclave. Ce vêtement a ainsi rempli une fonction symbolique, réassigner un homme, il n'assume plus aucune fonction de protection.

\section{Étoffes venues d'au-delà des eaux}

15 Le parcours interculturel que propose La Saison de l'ombre, sous sa forme de "polar historique » (Mokam 2015), ne se limite pas au face-à-face mulongo / bwele, et, s'agissant du vêtement, à l'opposition battre/tisser. Assez tôt en effet dans le récit sont mentionnés de mystérieux étrangers qui possèdent des étoffes inconnues; ainsi dans les propos de Bwemba, ce chasseur bwele qui conduira Mutango sur son territoire:

Le chasseur hausse les épaules. Son peuple connaît bien celui de la côte. Ils sont voisins. Ceux qui vivent sur les limites du monde connu sont, d'après lui, terriblement prétentieux. Depuis qu'ils ont rencontré les étrangers venus par les eaux, ils se croient les égaux du divin. Leurs nouveaux amis les fournissent en étoffes inconnues dans cette partie de misipo. Ils leur donnent aussi des armes, des bijoux et des choses qu'on ne saurait nommer. (Miano 2013 : 79)

Avant que Mutango ne découvre les usages bwele, Bwemba, qui le conduit chez les siens, introduit dans le récit une dimension de profondeur anthropologique : les Mulongo, les Bwele (« Son peuple »), les Côtiers, ainsi qu'ils seront désignés dans la suite du récit, et « les étrangers venus par les eaux ». Or, si les Bwele savent tisser de fines étoffes, et en offrir à leurs hôtes, les étranges étrangers à l'autre bout de la chaîne de voisinage et 
d'amitié en donnent ou en vendent d'autres, plus fines encore peut-être, mais surtout plus étonnantes, assorties à des objets ou corrélées à des gestes inattendus. C'est qu'en elles ça n'est plus la fonction de protection qui prévaut, ni même une fonction sociosymbolique, mais leur puissance esthétique ${ }^{15}$, qui leur confère un pouvoir sur les âmes.

Ce n'est qu'à la fin du roman qu'Eyabe entendra elle aussi parler de ces étoffes inconnues dans le récit de Mukudi, le jeune mulongo qu'elle a reconnu et qui a perdu son nom avec sa liberté :

Les Côtiers avaient rapidement pris conscience des avantages à tirer de relations avec les hommes aux pieds de poule. Ces derniers leur procuraient des marchandises étonnantes contre de l'huile, des dents ou des défenses d'éléphants. Ils avaient été les premiers à se vêtir d'étoffes tissées par les étrangers. Ces tissus à motifs imprimés faisaient fureur, chez les Isedu. Leurs princes étaient, par ailleurs, entrés en possession d'armes qui crachaient la foudre, faisaient un bruit de tonnerre. Quand leurs associés avaient réclamé des personnes humaines en échange de ces équipements, les Côtiers leur avaient d'abord remis quelques-uns de leurs soumis ou des individus ayant gravement contrevenu aux lois du clan. (Miano 2013 : 193-194)

Ce passage à la fin du roman livre à Eyabe la clé indirecte de la disparition des douze jeunes mâles mulongo, raison de son périple jusqu'à la Côte. Indice s'il en fallait que le vêtement sert de repère dans le récit, ces étrangers " aux pieds de poule " (il s'agit des Blancs, des Européens) sont désignés, non par la couleur de leur peau, mais par les étranges vêtements qui leur couvrent les jambes ${ }^{16}$. Or, ces visiteurs, venus de royaumes très éloignés, ont introduit un commerce glaçant : des étoffes imprimées et des armes à foudre et à tonnerre contre de l'huile, de l'ivoire ou des hommes. Les « motifs imprimés » renvoient à la chronologie de l'Occident, qui fabrique les indiennes à partir du XVII ${ }^{\mathrm{e}}$ siècle : la "fureur " qu'ils déclenchent, en Europe comme, dans ce récit, sur ces côtes africaines, manifeste que leur fonction esthétique est liée à un pouvoir à la fois mental et géopolitique. Elle occulte voire contredit la fonction initiale de protection.

Les disparus, parmi lesquels le fils d'Eyabe, auront ainsi pu être vendus contre ces tissus ensorcelants. La suite du récit de Mukudi livre d'autres indices accablants :

Dédaignant les mabato en fibres ou en écorce, les femmes de haut rang n'arboraient plus que des étoffes imprimées, dont on disait que les étrangers aux pieds de poule les créaient exclusivement pour le plaisir des dignitaires isedu. On constatait, d'ailleurs, qu'ils ne portaient pas eux-mêmes ces tissus bariolés. (Miano 2013: 199-200)

Eyabe, femme mulongo qui entend ce récit au terme de son parcours, ne peut qu'y voir un renversement plus radical que l'inversion femme/homme qui choquait Mutango chez les Bwele dans l'activité de tissage. Les femmes nobles renoncent à la protection de l'écorce et de la forêt, pour livrer leurs corps à des tissus inconnus, imprimés, porteurs de figures étrangères, tissus dangereux assurément puisque ceux qui les fournissent ne s'en vêtent pas. La fonction de protection de l'étoffe s'inverse donc ici, au bénéfice du "plaisir », de la fonction esthétique. Cette côte est vraiment lieu autant que signe de la fin du monde. Parvenue à ce point, Eyabe (et le lecteur à travers elle) peut pressentir ou anticiper la fin de ce monde en soi que fut la communauté mulongo.

Sans que Mutango y prête attention, fasciné qu'il était par la mention des armes à feu, le chasseur Bwemba lui avait révélé, évoquant ces mêmes «étrangers venus par les eaux » :

On dit que ces étrangers sont les émissaires de lointains dignitaires, désireux de s'allier avec leurs homologues, de ce côté-ci de la Création. Pour faire la preuve de leurs bonnes intentions, ils ont couvert les princes côtiers de présents, raison pour 
laquelle ces derniers se disent désormais leurs frères, les hébergent dans leurs concessions.

Cela fait déjà un moment que leur embarcation, une immense pirogue bardée d'étoffes destinées à emprisonner le souffle du vent, mouille au large du pays côtier. (Miano 2013 : 80-81)

Pour les Mulongo, la peau ou l'écorce protège le vivant, arbre ou fauve. Le vêtement que les femmes en tirent protège, pour peu qu'elles battent la fibre ou l'écorce, animant ainsi le végétal. Vêtir, pour un Mulongo, c'est donc d'abord protéger. Or, Mutango découvrira de ses yeux, sans réellement le comprendre, qu'on peut vêtir la terre, la couvrir d'étoffe, comme si la terre immobile elle-même requérait protection. Si c'en est une, cette protection s'assortira déjà, dans l'esprit de Mutango, de lourdes menaces. Mais, dans le détail du récit de Bwemba, il aurait dû prêter attention à mieux, ou pire : ces étrangers ont revêtu d'étoffes une pirogue pour "emprisonner le souffle du vent ». La subversion est ici totale. Si vêtir la terre semble aussi absurde que menaçant, comment et pourquoi, à quel prix vêt-on le vent?

Vêtir ici n'est plus protéger, mais tout au contraire prendre, capturer, emprisonner. Et de même que la voile innommée entend non pas protéger mais profiter du souffle et de l'énergie du vent, de même les étoffes imprimées, venues d'au-delà des eaux, entendent capturer le souffle et le désir de l'homme pour l'enfermer dans l'offre et la demande. Le désir des femmes en particulier, délaissant la force de leur parole collective et créatrice - au moins dans le monde mulongo-, sera ainsi excité et capturé dans ce marché de l'étoffe, comme le vent moteur dans la voile. L'étoffe, loin de protéger, devient ainsi le premier maillon d'une chaîne commerciale dont, par-delà les eaux, des " marchandise [s] » humaines (Colbert 1665 : article 7) seront les derniers. La fonction de protection du vêtement s'est inversée, sa fonction symbolique s'est brouillée, sa fonction esthétique domine alors mais pour mieux cacher son rôle d'appât au bout de la chaîne de domination, appât que lance une communauté naturaliste prédatrice ${ }^{17}$ à d'autres, analogiques, sans égard ni regard pour leurs ontologies propres. Et c'est ainsi que l'homme devient fongible, semblable à ce qui le vêt.

\section{Conclusion}

À l'issue de ce parcours en trois étapes - mulongo, bwele, européenne - des symboliques interculturelles du vêtement dans La Saison de l'ombre, nous souhaitons mettre en exergue quelques lignes parmi les dernières du roman :

Peu à peu, l'eau a commencé à rendre des effets : les sagaies, les amulettes des soldats mulongo; le mpondo du chef, celle de ses mbondi que la tourbe avait emprisonnée - l'autre ayant été retrouvée plus tard par Ebeise - son ekongo et son bâton d'autorité. Le tout sera remis à l'ancienne, en attendant l'érection du sanctuaire. (Miano $2013: 241$ )

Un monde a été anéanti. L'eau qui, tout au long du récit, de façon volontiers onirique, puis, lorsqu'Eyabe atteint la Côte, sur un mode plus réaliste, a figuré frontière et lieu de disparition, restitue ici, entre autres reliques éparses, le vêtement mulongo le plus prestigieux, le mpondo. De la peau du léopard, souverain de la forêt, les Mulongo avaient fait la cape royale de leur janea, leur chef souverain. C'est ce mpondo que Mutango, frère jaloux du chef, désirait revêtir, et c'est son désir désordonné qui l'aura conduit à sa perte. Or le janea Mukano, trompé par les Bwele, mort embourbé avec ses hommes dans les marais, n'aura pu comprendre, comme partiellement Mutango ou plus complètement 
Eyabe, les causes profondes du malheur de sa communauté. Causes terribles, ombre inouïe dont, sans crier gare, la saison s'est avancée trop vite. Car c'est à un prix terrible qu'on vêt la terre ou le vent. Or du désastre surnage un vêtement, symbole de souveraineté. Et, mémoire inclusive, porteur de toutes ces images, ce texte si puissant, savamment tissé.

« Instrument de la dignité », le vêtement révèle donc ici son contraire : sa fragilité, sa fongibilité désignent clairement celles de l'homme réduit au rang de marchandise et celles de communautés historiques détruites, désorganisées. Léonora Miano aura ainsi fourni une illustration littéraire puissante aux réflexions théoriques, historiques ou philosophiques contemporaines ${ }^{18}$ de la parution de son récit.

\section{BIBLIOGRAPHIE}

Bahuchet, Serge, 2009, «Pagne d'écorce », dans Y. Le Fur (dir.), Musée du quai Branly, la collection, Paris, Skira/Flammarion, p. 56-57, [En ligne], hal-00486921.

Balut, Pierre-Yves, 2014, Théorie du vêtement, Paris, L'Harmattan.

Bissa Enama, Patricia, 2014, «Léonora Miano ou la gynécocratie racontée dans La Saison de l'ombre ", dans A. D. Tang (dir.), L'CEuvre romanesque de Léonora Miano : fiction, mémoire et enjeux identitaires, Paris, L'Harmattan.

Chaulet Achour, Christiane, 2014, « La force du féminin dans La Saison de l'ombre (2013) », dans A. D. Tang (dir.), L'CEuvre romanesque de Léonora Miano : fiction, mémoire et enjeux identitaires, Paris, L'Harmattan.

Colbert, Jean-Baptiste, 1665, Code Noir, ou recueil d'édits, déclarations et arrêts concernant les Esclaves Nègres de l'Amérique, 1685, http://www.axl.cefan.ulaval.ca/amsudant/guyanefr1685.htm, consulté le 15 juillet 2019.

Coquery-Vidrovitch, Catherine, [1994] 2013, Les Africaines, Paris, Desjonquères.

Coquery-Vidrovitch, Catherine, 2011, Petite histoire de l'Afrique, Paris, La Découverte.

Coquery-Vidrovitch, Catherine et Mesnard, Éric, 2013, Être esclave - Afrique-Amériques, $X V^{e}-X I X^{e}$ siècle, Paris, La Découverte.

Delaporte, Yves, 1990, « Le vêtement dans les sociétés traditionnelles », dans J. Poirier (dir.), Histoire des mœurs I, vol. 2, Paris, Gallimard.

Depaule, Jean-Charles, 1990, « Le vêtement comme métaphore ? ", Égypte/Monde arabe, Première série, $n^{\circ} 3$, [En ligne], http://journals.openedition.org/ema/228, consulté le 15 juillet 2019.

DOI : $10.4000 /$ ema.228

Descola, Philippe, 2005, Par-delà nature et culture, Paris, Gallimard.

Du Bois, William Edward Burghardt, 1903, The Souls of Black Folk, Chicago, A. C. McClurg \& Co.

Gilroy, Paul, 1993, The Black Atlantic. Modernity and Double Consciousness, Londres, New York, Verso.

Grenouilleau, Olivier, [1997] 2018, La Traite des Noirs, Paris, PUF. 
Leroi-Gourhan, André, 1945, Milieu et techniques, Paris, Albin Michel.

Mbembe, Achille, 2013, Critique de la raison nègre, Paris, La Découverte.

Mokam, Yvonne-Marie, 2015, « Polar historique et mémoire de la capture des esclaves », Revue critique de fixxion française contemporaine, [En ligne], http://www.revue-critique-de-fixxionfrancaise-contemporaine.org/rcffc/article/view/fx10.08, consulté le 15 juillet 2019.

Tang, Alice Delphine (dir.), 2014, L'Euvre romanesque de Léonora Miano : fiction, mémoire et enjeux identitaires, Paris, L'Harmattan.

\section{Corpus}

Kourouma, Ahmadou, 1998, En attendant le vote des bêtes sauvages, Paris, Seuil.

Miano, Léonora, 2013, La Saison de l’ombre, Paris, Grasset \& Fasquelle.

\section{NOTES}

1. Le nom de ce pays, d'origine coloniale comme on sait, n'est jamais cité dans l'œuvre de Léonora Miano, quitte à ce qu'elle invente un nom de territoire fictif qui signifie «le pays ». Dans La Saison de l'ombre, l'absence de ce nom est parfaitement cohérente avec l'histoire et le point de vue interne mulongo retenu.

2. Notamment Du Bois (1903) ou Gilroy (1993).

3. Nous nous référons aux travaux classiques de Leroi-Gourhan (1945), Delaporte (1990), Depaule (1990) ainsi que, plus récemment, à ceux de Descola (2005) pour sa typologie des ontologies, Bahuchet (2009), notice sur un pagne d'écorce mbuti, Balut (2014), archéoanthropologue qui distingue notamment les fonctions abri et habit.

4. S'agissant du vêtement des sociétés traditionnelles, cette tripartition est notamment développée par Delaporte (1990 : 964-1012).

5. Notamment à travers sa réflexion sur l'histoire et le «devenir-nègre du monde » (Mbembe 2013).

6. C'est-à-dire de l'esclave historiquement associé à une couleur (niger). Ce terme est logiquement absent du récit de Léonora Miano du fait du point de vue empathique privilégié.

7. Ce thème du "déni de [la] mémoire» des mères de victimes de la traite est particulièrement mis en valeur dans l'article de Patricia Bissa Enama, « Léonora Miano ou la gynécocratie racontée dans La Saison de l'ombre » (2014).

8. Nous renvoyons à l'analyse de ce rôle et de cette force des femmes que propose Christiane Chaulet Achour, dans sa contribution «La force du féminin dans La Saison de l'ombre (2013)» (2014).

9. Rappelons que l'étymologie du pronom « on » est un substantif, « l'homme», porteur en français d'une signification souvent ambigüe, entre universel et masculin. En choisissant d'ailleurs d'utiliser la forme conservatrice «l'on", l'auteur remotive cette étymologie.

10. Pour Descola (2006), l'ontologie naturaliste (celle de l'Occident par exemple) a en commun avec l'ontologie analogique (propre aux sociétés traditionnelles de l'Afrique de 
l'Ouest) une différence des intériorités, ici homme/végétal. En revanche, elles se distinguent en ce que la première postule une « ressemblance des physicalités », quand la seconde suppose une « différence des physicalités ».

11. À l'inverse des femmes, que caractérisent silence, force intérieure et résilience face aux épreuves, les personnages masculins de l'œuvre, à rebours des schémas phallocentriques majoritaires dans les sociétés européennes ou africaines, sont volontiers associés au(x) défaut(s), à la perte. Qu'il s'agisse de disparition (les douze fils enlevés à la communauté), de bassesse d'âme (Mutango), d'incapacité à comprendre ou à décider (le noble chef Mukano perdu dans les marais, figure littéraire de ses atermoiements intérieurs), d'agonie (l'ancien Mutimbo) ou d'esclavage (Mukudi qui, à la fin du récit, refuse ce nom que lui donne Eyabe qui le reconnaît). Léonora Miano suggère ainsi par petites touches, au sein de cette communauté traditionnelle, une profonde impuissance des hommes à vouloir, à agir ou à (se) protéger. Mutango illustre cette incapacité d'abord morale des hommes jusqu'à la caricature.

12. Notamment par exemple dans le fameux passage où est évoquée la nudité des "paléos» (Kourouma 1998 : 12-20).

13. On compte en effet encore aujourd'hui 250 à 300 ethnies distinctes au Cameroun.

14. Voir supra, premier paragraphe, et note 2 .

15. Delaporte (1990: 1013), insiste sur les relations d'antagonisme qui existent entre les trois fonctions du vêtement des sociétés traditionnelles, et singulièrement entre la fonction esthétique et les fonctions pratiques.

16. Nous renvoyons au long échange explicatif entre Bwemba et Mutango, qui introduit l'expression - donnée par le peuple de la côte - pour désigner ces étrangers. Bwemba qui alors dit avoir vu ces hommes sans toutefois les approcher ne mentionne pas du tout leur couleur de peau mais, pour répondre à la perplexité de Mutango devant cette expression singulière, il décrit leur habillement qui les fait ressembler aux poulets (Miano 2013: 80). L'auteur a ainsi souligné, dans le texte comme dans des commentaires qu'elle a pu en faire, cette perception non raciale, en tout cas non épidermique, de l'Européen par les communautés africaines.

17. Selon Descola (2005), l'ontologie naturaliste qui caractérise l'Occident assume la ressemblance des physicalités (et la divergence des intériorités), ce qui lui permet de penser la continuité absolue des espèces vivantes. Celle en particulier, de l'animal au «nègre » dont il a fait historiquement le synonyme d'esclave : voir Coquery-Vidrovitch (2011: 13) ou Mbembe (2013: 34), dans sa critique de Buffon.

18. En particulier celles d'Achille Mbembe (2013), ou celles, plus exclusivement historiques, sur la condition d'esclave, que renouvellent Coquery-Vidrovitch et Mesnard (2013). 


\section{RÉSUMÉS}

Comment le vêtement est-il perçu dans La Saison de l'ombre (Léonora Miano, 2013) ? À travers ce référent d'apparence superficielle, le récit nous fait percevoir la profondeur interculturelle des échanges qui se tissent à l'époque de la traite négrière, cette "saison de l'ombre » des sociétés africaines. Les différentes fonctions anthropologiques du vêtement, mises tour à tour en valeur, accompagnent et signifient dans cette fiction le processus historique de réification et d'assignation au fongible de l'homme lui-même. Empathique, le récit nous rend solidaire de la communauté mulongo, victime dès l'ouverture d'un incendie et de disparitions inexplicables. Pour cette communauté, le vêtement, tiré de la forêt, protège comme elle. Toute étoffe, peau ou écorce battue, sert à vêtir, à protéger. Ce vêtement est l'affaire des femmes, volontiers associé à leur parole, à leur travail et signe de leur force que souligne l'auteur par volonté d'hommage. Mais le récit rend également compte de l'étonnement d'un personnage mulongo face à l'art du tissage de ses voisins bwele, et des usages étranges, porteurs de menaces, qui sont chez eux associés à ces tissus, confectionnés par les hommes, vêtant parfois non le corps, mais la terre. Plus loin encore, sur la côte, fin du monde inouïe pour les Mulongo des forêts, d'autres étoffes étrangères, plus dangereuses encore, emprisonnent le vent lui-même et bientôt le désir d'élégance des femmes comme la soif de pouvoir des hommes. Telles sont les frontières mentales et symboliques, culturelles et historiques qu'aura franchies le lecteur au long de ce récit en suivant l'aspect des étoffes à travers des yeux mulongo.

How are clothing and garment perceived in La Saison de l'ombre (Léonora Miano, 2013)? Through these superficial referents, the narrative makes the reader perceive the intercultural depth of exchanges woven in the early days of the slave trade, the "season of the shadow" for African societies. In this fiction the various anthropological functions of the garment, which are in turn foregrounded, accompany and signify the historical process of reification and assignment to the fungible of man himself. Told empathically, the story makes the reader identify with the Mulongo community, who, very early in the novel, fall victim to a fire and inexplicable disappearances. For this community, clothing, taken from the forest, protects as the forest does. Any cloth, skin or beaten bark is used to clothe and to protect. Clothing is a women's matter and is strongly associated with their voices, their work and presented as a sign of their strength by the author. But the story also recounts the astonishment of a Mulongo character when faced with his Bwele neighbors' art of weaving, and with the strange and threatening customs associated with their fabrics, woven by men and sometimes used to cover the earth rather than bodies. Further still, on the coast, the unimaginable end of the world for forest Mulongos, even more dangerous foreign fabrics are able to imprison the wind itself as well as women's desire for elegance and men's thirst for power. These are the mental and symbolic, cultural and historical boundaries that the reader will have crossed throughout the story by looking at fabrics through Mulongo eyes. 
INDEX

Keywords : stuff, clothing, Africa, story, focus, ontology, protection, semantics, aesthetics, Negro, fungible, Miano (Léonora)

Mots-clés : étoffe, vêtement, Afrique, récit, focalisation, ontologie, protection, sémantique, esthétique, nègre, fongible, Miano (Léonora)

\section{AUTEUR}

PIERRE-YVES DUFEU

Aix-Marseille Université 